\title{
USTC-ICTS-05-06
}

\section{Diquarks in Nonaquark States}

\author{
Gui-Jun Ding*, Mu-Lin Yan ${ }^{\dagger}$ \\ Interdisciplinary Center for Theoretical Study, \\ University of Science and Technology of China, \\ Hefei, Anhui 230026, China
}

\begin{abstract}
We study the nonaquark states $S^{0}(3115)$ and $S^{+}(3140)$ which are reported by KEK-PS (Phys.Lett. B597 (2004) 236; nucl-ex/0310018) by means of the quark model with diquark correlation. The nonaquark states form $\mathbf{1}, \mathbf{8}, \mathbf{1 0}, \overline{\mathbf{1 0}}, \mathbf{2 7}, \overline{\mathbf{3 5}} S U(3)$ multiplets. The flavor wave functions of all the nonaquark states are constructed through the standard tensor technique. The mass spectrum is studied by using Gell-Mann-Okubo mass formula. Some nonaquark mass sum rules are obtained. We further investigate the decay of $S^{0}(3115)$ and $S^{+}(3140)$ under the assumption of "fall-apart" mechanism. It has been found that the main decay mode is $\Sigma N N$ rather than $\Lambda N N$ which is consistent with experiment. Also we have uniquely determine the flavor wave function of $S^{0}(3115)$ which belong to 27-plet with the quantum number $Y=2, I=1, I_{z}=-1$. Whereas the exotic states $S^{+}(3140)$ can belong to either 27 -plet or $\overline{\mathbf{3 5}}$-plet. In the exact $S U(3)^{\text {flavor }} \times S U(3)^{\text {color }} \times S U(2)^{\text {spin }}$ limit, both $S^{0}(3115)$ and $S^{+}(3140)$ belong to 27-plet with negative parity. We predict that its flavor structure can be determined by measuring the branch fractions of its decay channels. The experiments to check this prediction are expected.
\end{abstract}

\footnotetext{
* e-mail address: dinggj@mail.ustc.edu.cn

$\dagger$ e-mail address: mlyan@ustc.edu.cn; corresponding author.
} 


\section{INTRODUCTION}

There is growing interest in exotic hadrons, which may open new windows for understanding the hadronic structures and QCD at low energies. Recently, the KEK-PS reported an tribaryon state $S^{0}(3115)$ [] in the reaction

$$
K^{-}+{ }^{4} H e \rightarrow S^{0}+p
$$

The mass of the state is $3117_{-4.4}^{+1.5} \mathrm{MeV}$, the decay width $\Gamma_{S^{0}}<21 \mathrm{MeV}$, and the main decay mode is $\Sigma N N$ rather than $\Lambda N N$. The peak in the proton spectrum is over the background with a significance level $13 \sigma$. A strange tribaryon $S^{+}(3140)$ of charge +1 was also reported in the reaction $K^{-}+{ }^{4} H e \rightarrow S^{+}+n$ [2]. The mass and decay width of this exotic state are $M_{S^{+}}=3141 \pm 3$ (stat. $)_{-1}^{+4}$ (sys.) $\mathrm{MeV}$ and $\Gamma_{S^{+}} \leq 23 \mathrm{MeV}$, which is about $25 \mathrm{MeV}$ higher than $S^{+}(3115)$, and its significance is $3.7 \sigma$. It also dominantly decay into $\Sigma N N$ rather than $\Lambda N N$.

The $S^{0}(3115)$ was first predicted by Akaishi and Yamazaki [3] as a deeply-bound kaonic state. Since the discovery of $S^{0}(3115)$ and $S^{+}(3140)$, there has been some theoretical discussion [4, 5, 6], in Ref [4] these exotic states are mainly analysized from MIT bag model, and they are identified as kaonic bound state in Ref [5, 6]. Since the quark dynamics could be regarded as a cornerstone for hadron physics, it is interesting to investigate the nonaquark states by means of the quark models. Various quark models have been used and proposed in studying the pentaquark baryon state [7, 8, 13]. Here we draw the spirit of Jaffe- Wilczek's work[7], since there are evidences for strong diquark correlation in the baryon spectrum 9], and, especially, in the light nonet-scalar $\left(J^{P C}=0^{++}\right)$meson spectrum. Their masses are generally below $1000 \mathrm{MeV}\left(f_{0}(600), f_{0}(980), a_{0}(980), \kappa(800)\right)$, and they do not favor the predictions of the $q \bar{q}$-models, but favor the diquark-antidiquark's quite well. Diquark is a boson with color $\overline{3}_{c}$, flavor $\overline{3}_{\mathrm{f}}$, and spin zero. Diquark correlation is also the basis of color superconductivity in dense quark matter which has not being observed experimentally. This configuration is favored by one gluon exchange [21, 22] and by instanton interactions [23, 24]. It may play important role in the exotic hadron physics. In this paper we try to investigate nonaquark baryons by means of diquarks model, and to learn what happens in the nonaquark case due to the strong diquark correlation. Meanwhile in order to understand the decay of nonaquark states, we suggest a decay mechanism which can qualitatively explain the experiments and give us new predictions. This decay mechanism is quite intuitive. To 
understand the structure of nonaquark, its mass spectrum and the decay mechanism are the main aims of this paper.

The paper is organized as follows, in the section II we study the direct products of two diquarks states, four diquarks states, and of four diquarks's plus one quark's. The irreducible tensors of the allowed nonaquark states are derived. The flavor wave functions are given by identifying the $S U(3)$ tensors with the physical tribaryon states. In Section III, the mass spectrum is derived by using the Gell-Mann-Okubo mass formula. The Section IV devotes to study the decays of $S^{0}(3115)$ and $S^{+}(3140)$ under the assumption that the decays caused by a "fall-apart" mechanism. We find when $S^{0}(3115)$ only belongs to a certain 27 -plet, its main decay mode is $\Sigma N N$ rather than $\Lambda N N$, and $S^{+}(3140)$ can belongs to either 27-plet or $\overline{35}$-plet. In Section V, we briefly summary the results and give some discussions.

\section{THE FLAVOR WAVE FUNCTION OF NONAQUARK STATES}

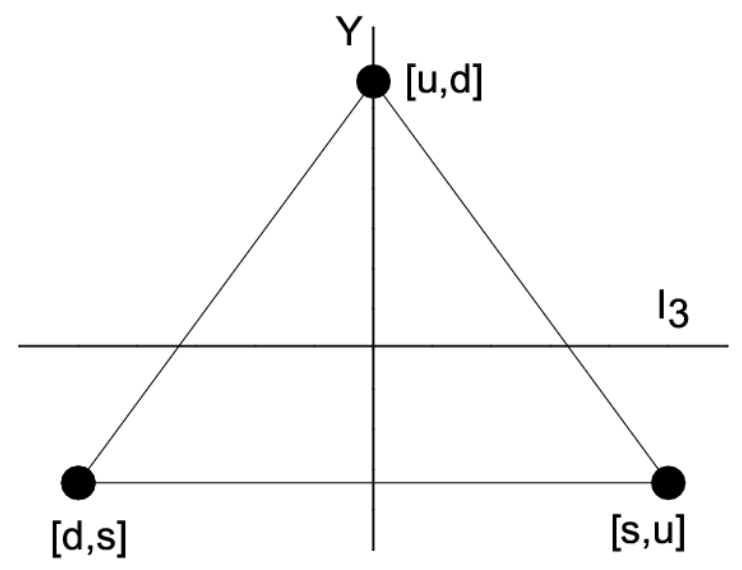

FIG. 1: $\overline{3}$ diquark .

Since the diquark is in the $\overline{3}_{\mathrm{f}}$, it has three configurations in flavor space, which are shown in Fig1. We denote them as

$$
\begin{aligned}
\mathbb{Q}^{1} & =\frac{1}{\sqrt{2}}[d, s] \\
\mathbb{Q}^{2} & =\frac{1}{\sqrt{2}}[s, u] \\
\mathbb{Q}^{3} & =\frac{1}{\sqrt{2}}[u, d]
\end{aligned}
$$




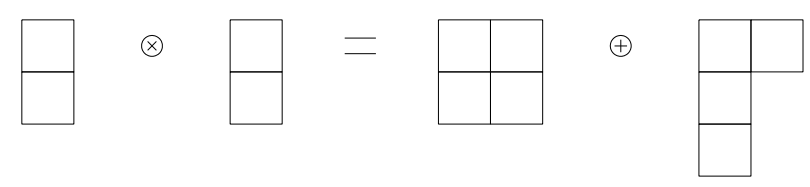

FIG. 2: direct product of two diquarks $\overline{\mathbf{3}} \otimes \overline{\mathbf{3}}=\overline{\mathbf{6}}+\mathbf{3}$.

where $u, d, s$ are respectively up quark,down quark and strange quark. It is obvious that there are following correspondences: $\mathbb{Q}^{1} \leftrightarrow \bar{u}, \mathbb{Q}^{2} \leftrightarrow \bar{d}, \mathbb{Q}^{3} \leftrightarrow \bar{s}$.

The tensors $T_{i_{1}, i_{2} \cdots i_{p}}^{j_{1}, j_{2}, \cdots j_{q}}$ which are the bases for irreducible representations of $S U(3)$ are totally symmetric to both all q upper indices and all p low indices, and also are traceless,

$$
\begin{aligned}
& T_{i_{1}, i_{2} \cdots i_{p}}^{j_{1}, j_{2} \cdots j_{q}}=T_{i_{1}, i_{2} \cdots i_{p}}^{j_{2}, j_{1} \cdots j_{q}}=T_{i_{2}, i_{1} \cdots i_{p}}^{j_{1}, j_{2}, \cdots j_{q}} \\
& T_{i_{1}, i_{2} \cdots i_{p}}^{i_{1}, j_{2} \cdots j_{q}}=0 .
\end{aligned}
$$

Since $\delta_{j}^{i}$, $\varepsilon^{i j k}$ and $\varepsilon_{\mathrm{ijk}}$ are tensors, we can use them to raise, low or contract indices when we construct new tensors that are bases of irreducible representation from the direct product tensor. The direct product of two diquarks is

$$
\mathbb{Q}^{i} \mathbb{Q}^{j}=\frac{1}{\sqrt{2}} S^{i j}+\frac{1}{2 \sqrt{2}} \varepsilon^{i j k} T_{k},
$$

with $S^{i j}=\frac{1}{\sqrt{2}}\left(\mathbb{Q}^{i} \mathbb{Q}^{j}+\mathbb{Q}^{j} \mathbb{Q}^{i}\right), A^{i j}=\frac{1}{\sqrt{2}}\left(\mathbb{Q}^{i} \mathbb{Q}^{j}-\mathbb{Q}^{j} \mathbb{Q}^{i}\right)$, and $T_{k}=\varepsilon_{i j k} A^{i j}$. So the decomposition of the direct product of two diquarks is $\overline{3} \otimes \overline{3}=\overline{6} \oplus 3$, which is shown in Fig2. in the Young tabular.

Since the two diquarks can be decomposed into $\overline{\mathbf{6}}$ plus 3 , the direct product of four diquarks raises $\overline{\mathbf{6}} \otimes \overline{\mathbf{6}}, \overline{\mathbf{6}} \otimes \mathbf{3}, \mathbf{3} \otimes \overline{\mathbf{6}}$ and $\mathbf{3} \otimes \mathbf{3}$. And the corresponding Young tabular is shown in Fig3. It is straightforward that

$$
\begin{aligned}
& \left(\mathbb{Q}^{i} \mathbb{Q}^{j}\right)\left(\mathbb{Q}^{m} \mathbb{Q}^{n}\right) \\
& =\frac{1}{2} S^{i j} S^{m n}+\frac{1}{4}\left(\varepsilon^{k i j} T_{k} S^{m n}+\varepsilon^{k m n} S^{i j} T_{k}\right)+\frac{1}{8} \varepsilon^{k i j} \varepsilon^{l m n} T_{k} T_{l} \\
& =\frac{1}{2 \sqrt{6}} T^{i j m n}+\frac{1}{4 \sqrt{2}}\left(\varepsilon^{a j m} \delta_{b}^{n} \delta_{c}^{i}+\varepsilon^{a i n} \delta_{b}^{m} \delta_{c}^{j}\right) S_{a}^{b c}+\frac{1}{2 \sqrt{6}}\left(\varepsilon^{a i m} \varepsilon^{b j n}+\varepsilon^{a j m} \varepsilon^{b i n}\right) T_{a b} \\
& +\frac{1}{4} \varepsilon^{k i j}\left[\tilde{T}_{k}^{m n}+\frac{1}{\sqrt{2}}\left(\delta_{k}^{m} \delta_{a}^{n}+\delta_{k}^{n} \delta_{a}^{m}\right) \tilde{Q}^{a}\right]+\frac{1}{4} \varepsilon^{k m n}\left[T_{k}^{i j}+\frac{1}{\sqrt{2}}\left(\delta_{k}^{i} \delta_{a}^{j}+\delta_{k}^{j} \delta_{a}^{i}\right) Q^{a}\right] \\
& +\frac{1}{4} \varepsilon^{k i j} \varepsilon^{l m n}\left(\sqrt{2} S_{k l}+\varepsilon_{k l a} T^{a}\right)
\end{aligned}
$$


( 1 )

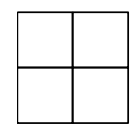

$\otimes$
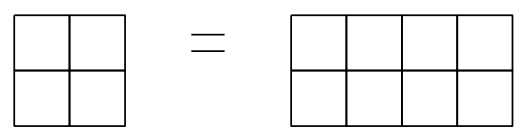

$\oplus$

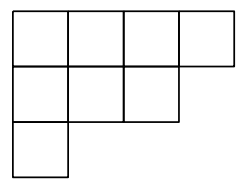

$\oplus$

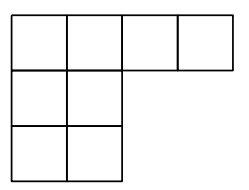

( II )

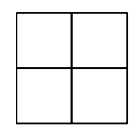

$\otimes$
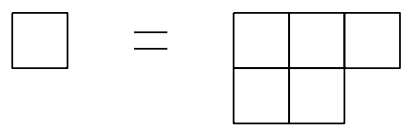

$\oplus$

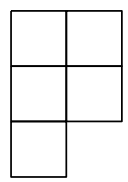

( III )

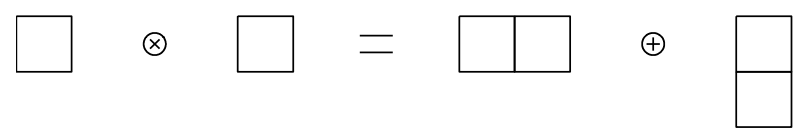

FIG. 3: The direct product of four diquarks: (I) $\overline{\mathbf{6}} \otimes \overline{\mathbf{6}}=\overline{\mathbf{1 5}}_{1}+\overline{\mathbf{1 5}}_{2}+\mathbf{6}$; (II) $\overline{\mathbf{6}} \otimes \mathbf{3}=\overline{\mathbf{1 5}}_{\mathbf{2}}+\overline{\mathbf{3}}$; (III) $\mathbf{3} \otimes \mathbf{3}=\mathbf{6} \oplus \overline{\mathbf{3}}$.

where the tensors in the above formula are defined as followings.

$$
\begin{aligned}
& T^{i}=\frac{1}{8} \varepsilon^{i j k}\left(T_{j} T_{k}-T_{k} T_{j}\right) \\
& Q^{i}=\frac{1}{\sqrt{8}} S^{i j} T_{j} \\
& \tilde{Q}^{i}=\frac{1}{\sqrt{8}} T_{j} S^{j i} \\
& S_{i j}=\frac{1}{4 \sqrt{2}}\left(T_{i} T_{j}+T_{j} T_{i}\right) \\
& T^{i j m n}=\frac{1}{\sqrt{6}}\left(S^{i j} S^{m n}+S^{m j} S^{i n}+S^{i n} S^{j m}+S^{m i} S^{j n}+S^{j n} S^{i m}+S^{m n} S^{i j}\right) \\
& S_{i}^{j k}=\frac{1}{\sqrt{2}} \varepsilon_{i m n}\left(S^{j m} S^{k n}+S^{k m} S^{j n}\right) \\
& \tilde{T}_{i}^{j k}=T_{i} S^{j k}-\frac{1}{\sqrt{2}}\left(\delta_{i}^{j} \delta_{m}^{k}+\delta_{i}^{k} \delta_{m}^{j}\right) \tilde{Q}^{m} \\
& T_{i}^{j k}=S^{j k} T_{i}-\frac{1}{\sqrt{2}}\left(\delta_{i}^{j} \delta_{m}^{k}+\delta_{i}^{k} \delta_{m}^{j}\right) Q^{m}
\end{aligned}
$$

So the four diquarks product can be decomposed into $\overline{\mathbf{1 5}}_{1} \oplus \overline{\mathbf{1 5}}_{2}(3) \oplus \overline{\mathbf{3}}(3) \oplus \mathbf{6}$, where the numbers in the parentheses denote the degeneracy in each multiplet. The tensors corresponding to $\overline{\mathbf{3}}$ are $T^{i}, Q^{i}, \tilde{Q}^{i}$, the tensor $S_{i j}$ form the bases of the irreducible representation $\mathbf{6}$, the tensor corresponding to $\overline{\mathbf{1 5}}_{1}$ is $T^{i j m n}$, and the tensors $S_{i}^{j k}, \tilde{T}_{i}^{j k}, T_{i}^{j k}$ are respectively the bases of the irreducible representation $\overline{\mathbf{1 5}}_{2}$. 
(I)

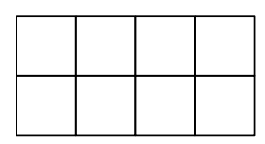

$\otimes$

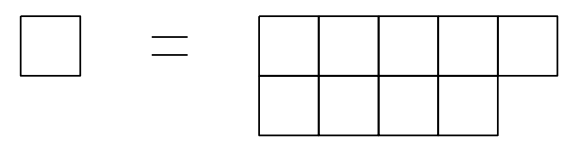

(II)

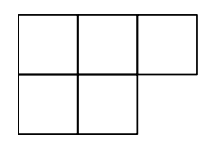

$\otimes$
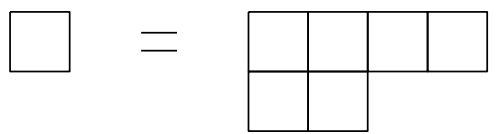

$\oplus$

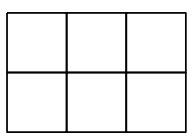

$\oplus$

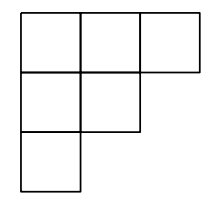

$\oplus$

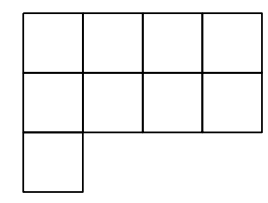

(III)

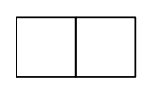

$\otimes$

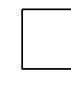

=

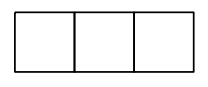

$\oplus$

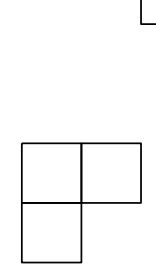

(IV)

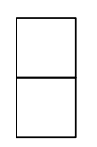

$\otimes$

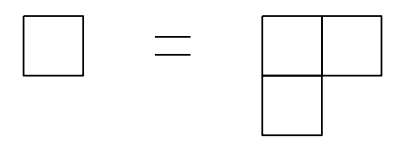

$\oplus$

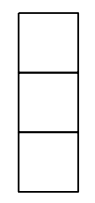

FIG. 4: direct product of four diquarks and a quark (I) $\overline{\mathbf{1 5}}_{1} \otimes \mathbf{3}=\overline{\mathbf{3 5}}+\overline{\mathbf{1 0}}$; (II) $\overline{\mathbf{1 5}}_{2} \otimes \mathbf{3}=\mathbf{2 7}+\overline{\mathbf{1 0}}+\mathbf{8}$; (III) $\mathbf{6} \otimes \mathbf{3}=\mathbf{1 0} \oplus \mathbf{8} ;(\mathrm{IV}) \overline{\mathbf{3}} \otimes \mathbf{3}=\mathbf{8} \oplus \mathbf{1}$.

\section{A. Nonaquark states}

Since quark is in the fundamental representation $\mathbf{3}$, when the four diquarks form the irreducible representation $\overline{\mathbf{3}}$, the nonaquark state must be in the representation $\overline{\mathbf{3}} \otimes \mathbf{3}=\mathbf{8}+\mathbf{1}$. This means the nonaquark state can either in the octet or in the singlet. We use $\mathcal{T}^{i}$ to stand for $T^{i}, Q^{i}, \tilde{Q}^{i}$, then the tensor product $\mathcal{T}^{i} q_{n}$ can be decomposed as follows

$$
\mathcal{T}^{i} q_{n}=\sqrt{2}\left(P_{n}^{i}+\frac{1}{\sqrt{3}} \delta_{n}^{i} S\right)
$$

where $S=\frac{1}{\sqrt{6}} \mathcal{T}^{m} q_{m}, P_{n}^{i}=\frac{1}{\sqrt{2}}\left(\mathcal{T}^{i} q_{n}-\sqrt{\frac{2}{3}} \delta_{n}^{i} S\right) . P_{n}^{i}$ stand for the nonaquark octet, and that $S$ stands for the nonaquark singlet.

When the four diquarks are in the representation $\overline{\mathbf{6}} \otimes \overline{\mathbf{6}}\left(=\overline{\mathbf{1 5}}_{1} \oplus \overline{\mathbf{1 5}}_{2} \oplus \mathbf{6}\right.$ (see Fig. 3)), they can form the irreducible representative of $\mathbf{6}$. Since $\mathbf{6} \otimes \mathbf{3}=\mathbf{1 0} \oplus \mathbf{8}$, the nonaquark 
states can be in decuplet or octet.

$$
S_{i j} q_{n}=\frac{1}{\sqrt{3}}\left[T_{i j k}+\varepsilon_{m j n} P_{i}^{m}+\varepsilon_{m i n} P_{j}^{m}\right]
$$

with $T_{i j k}=\frac{1}{\sqrt{3}}\left[S_{i j} q_{n}+S_{i n} q_{j}+S_{j n} q_{i}\right], P_{i}^{j}=\frac{1}{\sqrt{3}} \varepsilon^{j a b} S_{i a} q_{b}$ and $T_{i j k}, P_{i}^{j}$ respectively correspond to the nonaquark decuplet and octet.

Again, for the four-diquarks states in $\overline{\mathbf{6}} \otimes \overline{\mathbf{6}}$, they can also form $\overline{\mathbf{1 5}}_{1}$. The direct product $\overline{\mathbf{1 5}}_{1} \otimes \mathbf{3}$ can be reduced as follows

$$
T^{i j k l} q_{n}=T_{n}^{i j k l}+\frac{1}{\sqrt{6}}\left(\delta_{n}^{i} \delta_{b}^{j} \delta_{c}^{k} \delta_{d}^{l}+\delta_{n}^{j} \delta_{b}^{i} \delta_{c}^{k} \delta_{d}^{l}+\delta_{n}^{k} \delta_{b}^{i} \delta_{c}^{j} \delta_{d}^{l}+\delta_{n}^{l} \delta_{b}^{i} \delta_{c}^{j} \delta_{d}^{k}\right) D^{b c d}
$$

where $T_{n}^{i j k l}=T^{i j k l} q_{n}-\frac{1}{\sqrt{6}}\left(\delta_{n}^{i} D^{j k l}+\delta_{n}^{j} D^{i k l}+\delta_{n}^{l} D^{i j k}\right), \quad D^{i j k}=\frac{1}{\sqrt{6}} T^{i j k n} q_{n}, T_{n}^{i j k l}$ and $D^{i j k}$ respectively mean that the nonaquark state belong to $\overline{\mathbf{3 5}}$-plet and $\overline{\mathbf{1 0}}$-plet.

Finally, we consider the case of that the four-diquarks states form $\overline{\mathbf{1 5}}_{2}$. The $\overline{\mathbf{1 5}}_{2}-$ fourdiquarks states have three irreducible representatives: they can be in the product of $\overline{\mathbf{6}} \otimes$ $\mathbf{3}, \quad \mathbf{3} \otimes \overline{\mathbf{6}}$, or $\overline{\mathbf{6}} \otimes \overline{\mathbf{6}}$. The corresponding tensors are $S_{i}^{j k}, \tilde{T}_{i}^{j k}$ or $T_{i}^{j k}$ respectively. Using $\mathcal{T}_{i}^{j k}$ to denote each one of them, then we have

$$
\mathcal{T}_{i}^{j k} q_{n}=\sqrt{2} T_{i n}^{j k}+\sqrt{\frac{2}{3}} \varepsilon_{i n m} D^{m j k}+\frac{4}{\sqrt{15}}\left(\delta_{n}^{k} \delta_{m}^{j}+\delta_{m}^{k} \delta_{n}^{j}\right) P_{i}^{m}-\frac{1}{\sqrt{15}}\left(\delta_{i}^{k} \delta_{m}^{j}+\delta_{m}^{k} \delta_{i}^{j}\right) P_{n}^{m},
$$

where

$$
\begin{aligned}
P_{j}^{i} & =\frac{1}{\sqrt{15}} \mathcal{T}_{j}^{i k} q_{k} \\
D^{i j k} & =\frac{1}{24}\left(\varepsilon^{j a b} \mathcal{T}_{a}^{k m} q_{b}+\varepsilon^{k a b} \mathcal{T}_{a}^{j m} q_{b}+\varepsilon^{m a b} \mathcal{T}_{a}^{j k} q_{b}\right) \\
T_{i n}^{j k} & =\frac{1}{2 \sqrt{2}}\left(\mathcal{T}_{i}^{j k} q_{n}+\mathcal{T}_{n}^{j k} q_{i}\right)-\frac{\sqrt{30}}{20}\left(\delta_{i}^{k} P_{n}^{j}+\delta_{n}^{k} P_{i}^{j}+\delta_{i}^{j} P_{n}^{k}+\delta_{n}^{j} P_{i}^{k}\right) .
\end{aligned}
$$

$T_{i n}^{j k}$ represent the nonaquark $\overline{35}$-plet, and $D^{i j k}, P_{j}^{i}$ correspond to nonaquark $\overline{10}$-plet and octet. The Young tabular of the tensor decomposit ion are shown in Fig4.

Now we take the color symmetry $S U(3)^{\text {color }}$ into the account. When the $S U(3)^{\text {flavor }} \times$ $S U(3)^{\text {color }} \times S U(2)^{\text {spin }}$ serves as an exact symmetry, then due to the boson statistics the full combined $($ flavor $\times$ color $\times$ spin $\times$ space $)$ wave-functions of four diquarks states must be symmetric. Since the spin of the diquark is zero, the spin-wave-function is trivially to be symmetric. Thus, the $($ space $\times$ flavor $\times$ color $)$ wave function must be symmetric. Under this constraint, only two choices are available: 
1)The space-wave-functions are symmetric: In this case, the relative angular momentum $\ell$ is even and starts from $\ell=0$, and then the (flavor $\times$ color) wave-functions are symmetric. They must therefore belong to the $\overline{495}$ dimensional irreducible representation of $\mathrm{SU}(9) \supset$ $\mathrm{SU}(3)^{\text {flavor }} \times \mathrm{SU}(3)^{\text {color }}$. The reduction of the $\mathrm{SU}(9)$ irreducible representation $\overline{495}$ with respect to $\mathrm{SU}(3)^{\text {flavor }} \times \mathrm{SU}(3)^{\text {color }}[25]$ is

$$
\overline{495}=\left(\overline{15}_{1}, \overline{15}_{1}\right)+\left(\overline{15}_{2}, \overline{15}_{2}\right)+(\overline{3}, \overline{3})+(6,6) .
$$

We can see only the four diquarks's states (flavor, color $)=(\overline{3}, \overline{3})$ can combine with the ninth quark with $($ flavor, color $)=(3,3)$ to form a color singlet. Because $\overline{3} \otimes 3=8 \oplus 1$, there may exist nonaquark nonet with positive parity. But the states $S^{0}(3115)$ and $S^{+}(3140)$ can not belong to this nonet, because the hypercharge of these two states is $Y=2$, while the maximum of hypercharge of the nonet is 1 .

2) The space-wave-functions are antisymmetric: This means that the relative angular momentum $\ell$ is odd and starts from $\ell=1$, and then the (flavor $\times$ color) wave-functions are antisymmetric. And they must belong to the 126 dimensional irreducible representation of $\mathrm{SU}(9) \supset \mathrm{SU}(3)^{\text {flavor }} \times \mathrm{SU}(3)^{\text {color }}$. The reduction of the $\mathrm{SU}(9)$ irreducible representation 126 with respect to $\mathrm{SU}(3)^{\text {flavor }} \times \mathrm{SU}(3)^{\text {color }}$ is

$$
126=(6,6)+\left(\overline{15}_{2}, \overline{3}\right)+\left(\overline{3}, \overline{15}_{2}\right)
$$

So only when the four diquarks in the state (flavor, color $)=\left(\overline{15}_{2}, \overline{3}\right)$, they can combine with the ninth quark to form a color singlet hadron. Because $\overline{15}_{2} \otimes 3=27 \oplus \overline{10} \oplus 8$, the nonaquark can only in flavor multiplet 27-plet, $\overline{10}$-plet, 8-plet, and the nonaquark state can not be flavor 35-plet. This is a rigorous result when the flavor symmetry is exactly. Considering, however, that $S U(3)^{\text {flavor }}$ is an approximative symmetry which will lead to $S U(3)^{\text {flavor }} \times S U(3)^{\text {color }} \times S U(2)^{\text {spin }}$ to be approximate, so we can not completely rule out 35-plet.

From the quantum number of $S^{0}(3115)$ and $S^{+}(3140)$, they possibly belong to the 27 plet or $\overline{\mathbf{3 5}}$-plet. In the exact $S U(3)^{\text {flavor }} \times S U(3)^{\text {color }} \times S U(2)^{\text {spin }}$ limit, both $S^{0}(3115)$ and $S^{+}(3140)$ belong to 27 -plet whose lowest angular momentum is $\ell=1$ (they are $\mathrm{P}$-wave states), and the corresponding weight diagram is Fig6. The Fig7 is the weight diagram for 35-plet. In the Figures, we show the names of these exotic states, with the subscripts that are the representation-dimensions and the isospin of the particle. Their superscript is the charge of the state. 
In tensor representations, the number of lower indices of $T_{i_{1}, \ldots, i_{p}}^{j_{1}, \ldots, k_{q}}$ is $p$ and that of upper indices is $q$. Now we suppose that among its lower indices the numbers of 1,2 , and 3 are $p_{1}, p_{2}$, and $p_{3}$, respectively, and that among upper indices it has $q_{1} 1, q_{2} 2$, and $q_{3} 3$. Then we have $p_{1}+p_{2}+p_{3}=p$ and $q_{1}+q_{2}+q_{3}=q$. The irreducible tensor is an eigenstate of hypercharge $Y$ and the third component of isospin $I_{3}$ with the eigenvalues [12, 14, 15]

$$
\begin{aligned}
Y & =p_{1}-q_{1}+p_{2}-q_{2}-\frac{2}{3}(p-q) \\
I_{3} & =\frac{1}{2}\left(p_{1}-q_{1}\right)-\frac{1}{2}\left(p_{2}-q_{2}\right) .
\end{aligned}
$$

The charge of the particle is obtained from the Gell-Mann-Nishijima formula, $Q=I_{3}+Y / 2$. By this way, we can match the $\mathrm{SU}(3)$ tensors to the physical baryon states.

\section{B. The wavefunction of Nonaquark $\overline{27}$-plet}

It is straightforward to write out the wave functions of nonaquark $\overline{\mathbf{2 7}}$-plets in the flavor space by means of the irreducible representation tensors. The $S_{27,1}^{+}$and $S_{27,1}^{0}$ read

$$
\begin{gathered}
S_{27,1}^{+}=\frac{1}{\sqrt{2}} T_{12}^{33}=\frac{1}{4}\left(\mathcal{T}_{1}^{33} q_{2}+\mathcal{T}_{2}^{33} q_{1}\right) \\
S_{27,1}^{0}=\frac{1}{2} T_{22}^{33}=\frac{1}{2 \sqrt{2}} \mathcal{T}_{2}^{33} q_{2},
\end{gathered}
$$

where $\mathcal{T}_{k}^{i j}$ stands for the tensors of four-diquarks states. We now provide the explicit expressions of $S_{27,1}^{+}$and $S_{27,1}^{0}$ for the each irreducible representatives of $\overline{\mathbf{1 5}}_{2}$ in order:

1. The case of $\overline{\mathbf{1 5}}_{2} \subset \overline{\mathbf{6}} \otimes \overline{\mathbf{6}}$ : In this case $\mathcal{T}_{i}^{j k}$ is $S_{i}^{j k}$ which has been defined in Eq.(11). So,

$$
\begin{aligned}
& S_{1}^{33}=\frac{1}{2 \sqrt{2}}([u, d][s, u][u, d][u, d]+[s, u][u, d][u, d][u, d]-[u, d][u, d][u, d][s, u] \\
& -[u, d][u, d][s, u][u, d]), \\
& S_{2}^{33}=\frac{1}{2 \sqrt{2}}([u, d][u, d][u, d][d, s]+[u, d][u, d][d, s][u, d]-[u, d][d, s][u, d][u, d] \\
& -[d, s][u, d][u, d][u, d])
\end{aligned}
$$

the wave function of $S_{27_{1}, 1}^{+}$and $S_{27_{1}, 1}^{0}$ are

$$
S_{27_{1}, 1}^{+}=\frac{1}{4}\left(S_{1}^{33} q_{2}+S_{2}^{33} q_{1}\right)
$$




$$
\begin{aligned}
= & \frac{1}{8 \sqrt{2}}([u, d][s, u][u, d][u, d] d+[s, u][u, d][u, d][u, d] d-[u, d][u, d][u, d][s, u] d \\
& -[u, d][u, d][s, u][u, d] d+[u, d][u, d][u, d][d, s] u+[u, d][u, d][d, s][u, d] u \\
& -[u, d][d, s][u, d][u, d] u-[d, s][u, d][u, d][u, d] u) ; \\
S_{27_{1}, 1}^{0}= & \frac{1}{2 \sqrt{2}} S_{2}^{33} q_{2} \\
= & \frac{1}{8}([u, d][u, d][u, d][d, s] d+[u, d][u, d][d, s][u, d] d-[u, d][d, s][u, d][u, d] d \\
& -[d, s][u, d][u, d][u, d] d) .
\end{aligned}
$$

2. The case of $\overline{\mathbf{1 5}}_{2} \subset \mathbf{3} \otimes \overline{\mathbf{6}}$ : The $\mathcal{T}_{i}^{j k}$ is $\tilde{T}_{i}^{j k}$ which is defined in Eq.(12), and

$$
\begin{aligned}
& \tilde{T}_{1}^{33}=T_{1} S^{33}=\frac{1}{2}([s, u][u, d][u, d][u, d]-[u, d][s, u][u, d][u, d]) \\
& \tilde{T}_{2}^{33}=T_{2} S^{33}=\frac{1}{2}([u, d][d, s][u, d][u, d]-[d, s][u, d][u, d][u, d])
\end{aligned}
$$

then, the wave functions of $S_{27_{2}, 1}^{+}$and $S_{27_{2}, 1}^{0}$ are

$$
\begin{aligned}
S_{27_{2}, 1}^{+}= & \frac{1}{4}\left(\tilde{T}_{1}^{33} q_{2}+\tilde{T}_{2}^{33} q_{1}\right) \\
= & \frac{1}{8}([s, u][u, d][u, d][u, d] d-[u, d][s, u][u, d][u, d] d+[u, d][d, s][u, d][u, d] u \\
& -[d, s][u, d][u, d][u, d] u) \\
S_{27_{2}, 1}^{0}= & \frac{1}{2 \sqrt{2}} \tilde{T}_{2}^{33} q_{2}=\frac{1}{4 \sqrt{2}}([u, d][d, s][u, d][u, d] d-[d, s][u, d][u, d][u, d] d) .
\end{aligned}
$$

3. The case of $\overline{\mathbf{1 5}}_{2} \subset \overline{\mathbf{6}} \otimes \mathbf{3}$ : In this case $\mathcal{T}_{i}^{j k}$ is the tensor $T_{i}^{j k}$ defined in Eq.(13), obviously

$$
\begin{aligned}
& T_{1}^{33}=S^{33} T_{1}=\frac{1}{2}([u, d][u, d][s, u][u, d]-[u, d][u, d][u, d][s, u]) \\
& T_{2}^{33}=S^{33} T_{2}=\frac{1}{2}([u, d][u, d][u, d][d, s]-[u, d][u, d][d, s][u, d])
\end{aligned}
$$

The wave functions of the two states are

$$
\begin{aligned}
S_{27_{3}, 1}^{+}= & \frac{1}{4}\left(T_{1}^{33} q_{2}+T_{2}^{33} q_{1}\right) \\
= & \frac{1}{8}([u, d][u, d][s, u][u, d] d-[u, d][u, d][u, d][s, u] d+[u, d][u, d][u, d][d, s] u \\
& -[u, d][u, d][d, s][u, d] u) \\
S_{27_{3}, 1}^{0} & =\frac{1}{2 \sqrt{2}} T_{2}^{33} q_{2} \\
& =\frac{1}{4 \sqrt{2}}([u, d][u, d][u, d][d, s] d-[u, d][u, d][d, s][u, d] d)
\end{aligned}
$$




\section{The wave function of Nonaquark $\overline{35}$-plet}

Since $\overline{\mathbf{3 5}}$ can not be completely excluded (see the subsection II.A), we should also discuss it's wavefunction for completeness. It is easy to identify

$$
\begin{aligned}
& T_{2}^{1333}=-\sqrt{6} S_{\frac{0}{35,1},}^{0}, \quad T_{1}^{1333}=-\sqrt{3} S_{\frac{+}{35}, 1}^{+}-\sqrt{2} S_{\frac{+}{35}}^{+} \\
& T_{2}^{2333}=\sqrt{3} S_{\frac{+}{35}, 1}^{+}-\sqrt{2} S_{\frac{+}{35}}^{+}, \quad T_{3}^{3333}=2 \sqrt{2} S_{\frac{+}{35}}^{+}, \quad T_{1}^{2333}=-\sqrt{6} S_{35,1}^{++}
\end{aligned}
$$

then

$$
\left\{\begin{array}{l}
S_{\frac{0}{35,1}}=-\frac{1}{\sqrt{6}} T_{2}^{1333} \\
S_{\frac{+}{35,1}}^{+}=\frac{1}{2 \sqrt{3}}\left(T_{2}^{2333}-T_{1}^{1333}\right) \\
S_{\frac{+}{35}}^{+}=\frac{1}{2 \sqrt{2}} T_{3}^{3333}
\end{array}\right.
$$

and $T_{n}^{i j k l}$ is defined in Eq.(16). It is easy to see

$$
\begin{gathered}
T^{1333}=\frac{1}{\sqrt{6}}\left(S^{13} S^{23}+S^{33} S^{13}+S^{13} S^{33}+S^{31} S^{33}+S^{33} S^{13}+S^{33} S^{13}\right) \\
=\frac{3}{\sqrt{6}}\left(S^{13} S^{33}+S^{33} S^{13}\right) \\
=\frac{3}{4 \sqrt{6}}([d, s][u, d][u, d][u, d]+[u, d][d, s][u, d][u, d] \\
\quad+[u, d][u, d][d, s][u, d]+[u, d][u, d][u, d][d, s]) \\
T^{2333}=\frac{3}{\sqrt{6}}\left(S^{23} S^{33}+S^{33} S^{23}\right) \\
=\frac{3}{4 \sqrt{6}}([s, u][u, d][u, d][u, d]+[u, d][s, u][u, d][u, d] \\
\quad+[u, d][u, d][s, u][u, d]+[u, d][u, d][u, d][s, u]) \\
T^{3333}=\sqrt{6} S^{33} S^{33}=\frac{\sqrt{6}}{2}[u, d][u, d][u, d][u, d]
\end{gathered}
$$

and the wave function of $S_{35,1}^{0}, S_{\overline{35,1}}^{+}, S_{\frac{+}{35}}$ are as followings

$$
\begin{array}{r}
S_{\frac{0}{35,1}=}^{0}-\frac{1}{\sqrt{6}} T_{2}^{1333}=-\frac{1}{\sqrt{6}} T^{1333} q_{2} \\
=-\frac{1}{8}([d, s][u, d][u, d][u, d]+[u, d][d, s][u, d][u, d] \\
\\
+[u, d][u, d][d, s][u, d]+[u, d][u, d][u, d][d, s]) d \\
S_{\frac{15}{4}, 1}^{+}=\frac{1}{2 \sqrt{3}}\left(T_{2}^{2333}-T_{1}^{1333}\right)=\frac{1}{2 \sqrt{3}}\left(-T^{1333} q_{1}+T^{2333} q_{2}\right)
\end{array}
$$




$$
\begin{aligned}
= & \frac{1}{8 \sqrt{2}}(-[d, s][u, d][u, d][u, d] u-[u, d][d, s][u, d][u, d] u-[u, d][u, d][d, s][u, d] u \\
& -[u, d][u, d][u, d][d, s] u+[s, u][u, d][u, d][u, d] d+[u, d][s, u][u, d][u, d] d \\
& +[u, d][u, d][s, u][u, d] d+[u, d][u, d][u, d][s, u] d) \\
S_{\frac{+}{35}=} & \frac{1}{2 \sqrt{2}} T_{3}^{3333}=\frac{1}{6 \sqrt{2}}\left(-2 T^{1333} q_{1}-2 T^{2333} q_{2}+T^{3333} q_{3}\right) \\
= & -\frac{1}{8 \sqrt{3}}([d, s][u, d][u, d][u, d] u+[u, d][d, s][u, d][u, d] u+[u, d][u, d][d, s][u, d] u \\
& +[u, d][u, d][u, d][d, s] u+[s, u][u, d][u, d][u, d]+[u, d][s, u][u, d][u, d] \\
& +[u, d][u, d][s, u][u, d]+[u, d][u, d][u, d][s, u]-2[u, d][u, d][u, d][u, d] s)
\end{aligned}
$$

\section{THE MASS SPECTRUM OF NONAQUARK STATES}

Since all the particles belonging to an irreducible representation of $S U(3)$ are degenerate

in $S U(3)$ symmetry limit, it is necessary to introduce the $S U(3)$ symmetry breaking terms into the Hamiltonian in order to obtain the mass splitting. The Hamiltonian that breaks $S U(3)$ symmetry but still preserves the isospin symmetry and hypercharge is proportional to the Gell-Mann matrix $\lambda_{8}$, and the baryon mass can be obtained by constructing $S U(3)$ singlet term including the hypercharge tensor, in this way we obtain the Gell-Mann-Okubo mass formula:

$$
M=M_{0}+\alpha Y+\beta D_{3}^{3}
$$

where $M_{0}$ is a common mass of a given multiplet and $D_{3}^{3}=I(I+1)-\frac{Y^{2}}{4}-\frac{C}{6}$ with $C=2(p+q)+\frac{2}{3}\left(p^{2}+p q+q^{2}\right)$ for the $(\mathrm{p}, \mathrm{q})$ representation. $\alpha$ and $\beta$ are mass constant that are in principle different for different multiplets. Using these constants, we can obtain the masses of all the baryons within the multiplet. Note that in this picture the isospin is conserved.

\section{A. The mass spectrum of Nonaquark 27-plet}

In the case of 27-plet, $p=q=2$ and the corresponding weight diagram is Fig.(6). By using 
the Gell-Mann-Okubo mass formula Eq.(44) we can get all the masses of these states

$$
\begin{aligned}
& M_{S_{27,1}}=M_{27}+2 \alpha_{27}-\frac{5}{3} \beta_{27}, \quad M_{N_{27, \frac{3}{2}}}=M_{27}+\alpha_{27}+\frac{5}{6} \beta_{27}, \quad M_{N_{27, \frac{1}{2}}}=M_{27}+\alpha_{27}-\frac{13}{6} \beta_{27} \\
& M_{\Sigma_{27,2}}=M_{27}+\frac{10}{3} \beta_{27}, \quad M_{\Sigma_{27,1}}=M_{27}-\frac{2}{3} \beta_{27}, \quad M_{\Lambda_{27}}=M_{27}-\frac{8}{3} \beta_{27} \\
& M_{\Xi_{27, \frac{3}{2}}}=M_{27}-\alpha_{27}+\frac{5}{6} \beta_{27}, \quad M_{\Xi_{27, \frac{1}{2}}}=M_{27}-\alpha_{27}-\frac{13}{6} \beta_{27} \\
& M_{\Omega_{27,1}}=M_{27}-2 \alpha_{27}-\frac{5}{3} \beta_{27}
\end{aligned}
$$

The Gell-Mann-Okubo mass relation for 27-plet nonaquark baryons is

$$
3 M_{\Lambda_{27}}+M_{\Sigma_{27,1}}=2\left(M_{N_{27, \frac{1}{2}}}+M_{\Xi_{27, \frac{1}{2}}}\right)=4 M_{27}-\frac{26}{3} \beta_{27}
$$

The equal mass space relations also exist in the two separate sectors: $\left(\Omega_{27,1}, \Xi_{27, \frac{3}{2}}, \Sigma_{27,2}\right)$ and $\left(\Sigma_{27,2}, N_{27, \frac{3}{2}}, S_{27,1}\right)$. Their mass relations are as follows

$$
\begin{aligned}
& M_{\Omega_{27,1}}-M_{\Xi 27, \frac{3}{2}}=M_{\Xi 27, \frac{3}{2}}-M_{\Sigma_{27,2}}=-\alpha_{27}-\frac{5}{2} \beta_{27} \\
& M_{\Sigma_{27,2}}-M_{N_{27, \frac{3}{2}}}=M_{N_{27, \frac{3}{2}}}-M_{S_{27,1}}=-\alpha_{27}+\frac{5}{2} \beta_{27}
\end{aligned}
$$

From Eq.(45) we can further obtain some relation between mass of these states

$$
\begin{aligned}
& M_{\Lambda_{27}}+M_{S_{27,1}}=2 M_{N_{27, \frac{1}{2}}}, \quad M_{\Sigma_{27,2}}+M_{S_{27,1}}=2 M_{N_{27, \frac{3}{2}}}, 3 M_{\Sigma_{27,1}}+3 M_{S_{27,1}}=4 M_{N_{27, \frac{1}{2}}}+2 M_{N_{27, \frac{3}{2}}} \\
& 3 M_{\Xi_{27, \frac{3}{2}}}+6 M_{S_{27,1}}=5 M_{N_{27, \frac{1}{2}}}+4 M_{N_{27, \frac{3}{2}}}, \quad 3 M_{\Xi_{27, \frac{1}{2}}}+6 M_{S_{27,1}}=8 M_{N_{27, \frac{1}{2}}}+M_{N_{27, \frac{3}{2}}} \\
& 3 M_{\Omega_{27,1}}+9 M_{S_{27,1}}=10 M_{N_{27, \frac{1}{2}}}+2 M_{N_{27, \frac{3}{2}}}
\end{aligned}
$$

Both the masses of $\overline{\mathbf{3 5}}$-plets and the masses of 27-plets contain three parameters: $M_{\overline{35}}, \alpha_{\overline{35}}, \beta_{\overline{35}}$ or $M_{27}, \alpha_{27}, \beta_{27}$, but we only known experimentally the mass of $S^{0}(3115)$ and $S^{+}(3140)$. So we can not fix the masses of other nonaquark states which are predicted by us.

It mostly seems that $S^{0}(3115)$ and $S^{+}(3140)$ would belong to the same isospin multiplet and the mass difference between them are mainly due to electromagnetic interaction and the mass differences between u quark's and d quark's .

\section{B. The mass spectrum of the Nonaquark $\overline{35}$-plet}


By means of the Gell-Mann-Okubo mass formula eq.(44), and noting the $\overline{35}$-plet with $p=1, q=4$ whose weight diagram is shown in Fig7., the masses of all the exotic nonaquark states are as follows.

$$
\begin{aligned}
& M_{X_{\overline{35}, \frac{1}{2}}}=M_{\overline{35}}+3 \alpha_{\overline{35}}-\frac{11}{2} \beta_{\overline{35}}, \quad M_{S_{\overline{35}, 1}}=M_{\overline{35}}+2 \alpha_{\overline{35}}-3 \beta_{\overline{35}}, \quad M_{S_{\overline{35}}}=M_{\overline{35}}+2 \alpha_{\overline{35}}-5 \beta_{\overline{35}} \\
& M_{N_{\overline{35}, \frac{3}{2}}}=M_{\overline{35}}+\alpha_{\overline{35}}-\frac{\beta_{\overline{35}}}{2}, \quad M_{N_{\overline{35}, \frac{1}{2}}}=M_{\overline{35}}+\alpha_{\overline{35}}-\frac{7}{2} \beta_{\overline{35}}, \quad M_{\Sigma_{\overline{35}, 2}}=M_{0}+2 \beta_{\overline{35}} \\
& M_{\Sigma_{\overline{35}, 1}}=M_{\overline{35}}-2 \beta_{\overline{35}}, \quad M_{\Xi_{\overline{35}, \frac{5}{2}}}=M_{\overline{35}}-\alpha_{\overline{35}}+\frac{9}{2} \beta_{\overline{35}}, \quad M_{\Xi_{\overline{35}, \frac{3}{2}}}=M_{\overline{35}}-\alpha_{\overline{35}}-\frac{1}{2} \beta_{\overline{35}} \\
& M_{\Omega_{\overline{35}, 2}}=M_{\overline{35}}-2 \alpha_{\overline{35}}+\beta_{\overline{35}}
\end{aligned}
$$

We can find that the equal space rule holds for two sectors of nonaquark baryons: $\left(X_{\overline{35}, \frac{1}{2}}, S_{\overline{35}, 1}, N_{\overline{35}, \frac{3}{2}}, \Sigma_{\overline{35}, 2}, \Xi_{\overline{35}, \frac{5}{2}}\right)$ and $\left(S_{\overline{35}}, N_{\overline{35}, \frac{1}{2}}, \Sigma_{\overline{35}, 1}, \Xi_{\overline{35}, \frac{3}{2}}, \Omega_{\overline{35}, 2}\right)$. They satisfy the mass relations as follows

$$
\begin{aligned}
& M_{X_{\overline{35}, \frac{1}{2}}}-M_{S_{\overline{35}, 1}}=M_{S_{\overline{35}, 1}}-M_{N_{\overline{35}, \frac{3}{2}}}=M_{N_{\overline{35}, \frac{3}{2}}}-M_{\Sigma_{\overline{35}, 2}}=M_{\Sigma_{\overline{35}, 2}}-M_{\Xi_{\overline{35}, \frac{5}{2}}}=\alpha_{\overline{35}}-\frac{5}{2} \beta_{\overline{35}} \\
& M_{S_{\overline{35}}}-M_{N_{\overline{35}, \frac{1}{2}}}=M_{N_{\overline{35}, \frac{1}{2}}}-M_{\Sigma_{\overline{35}, 1}}=M_{\Sigma_{\overline{35}, 1}}-M_{\Xi_{\overline{35}, \frac{3}{2}}}=M_{\Xi_{\overline{35}, \frac{3}{2}}}-M_{\Omega_{\overline{35}, 2}}=\alpha_{\overline{35}}-\frac{3}{2} \beta_{\overline{35}}
\end{aligned}
$$

And we can also derive the mass relation between these nonaquark states

$$
\begin{aligned}
& M_{X_{\overline{35}, \frac{1}{2}}}+M_{N_{\overline{35}, \frac{3}{2}}}=2 M_{S_{\overline{35}, 1}}, \quad 3\left(M_{S_{\overline{35}}}-M_{S_{\overline{35}, 1}}\right)=2\left(M_{N_{\overline{35}, \frac{1}{2}}}-M_{N_{\overline{35}, \frac{3}{2}}}\right) \\
& M_{\Sigma_{\overline{35}, 2}}+M_{S_{\overline{35}, 1}}=2 M_{N_{\overline{35}, \frac{3}{2}}}, \quad M_{\Sigma_{\overline{35}, 1}}+3 M_{S_{\overline{35}, 1}}=2\left(M_{S_{\overline{35}}}+M_{N_{\overline{35}, \frac{3}{2}}}\right) \\
& M_{\Xi_{\overline{35}, \frac{5}{2}}}+2 M_{S_{\overline{35}, 1}}=3 M_{N_{\overline{35}, \frac{3}{2}}}, \quad 2 M_{\Xi_{\overline{35}, \frac{3}{2}}}+9 M_{S_{\overline{35}, 1}}=5 M_{S_{\overline{35}}}+6 M_{N_{\overline{35}, \frac{3}{2}}} \\
& M_{\Omega_{\overline{35}, 2}}+6 M_{S_{\overline{35}, 1}}=3 M_{S_{\overline{35}}}+4 M_{N_{\overline{35}, \frac{3}{2}}}
\end{aligned}
$$

\section{DECAY OF THE NONAQUARK STATES}

We assume the nonaquark state decays arise by a "fall-apart" mechanism [11, 16, 17, 18] without need for gluon exchange to trigger the decay. There are some discussions on this mechanism in the pentaquark spectrum studies. By this mechanism, a diquark in the pentaquark must be so clever that its two quarks are detached to two isolated quarks, and one of them enters into the adjacent diquarks to form a baryon separatively, and another combines with the residual anti-quark to form a meson, and then a pentaquark baryon decays into a usual baryon plus a meson. In this mechanism, the dynamics from the color coupling contributes a common factor to the decay amplitude for a certain flavor multiplet 
which is irrelevant to the discussions on its decay branch fractions. Extending this "fallapart" mechanism to nonaquark state decays is natural and straightforward: 1, one diquark is detached into two quarks; 2, these two quarks enter the adjacent diquaks, and form two baryons separatively; 3, the ninth quark also enter a diquark to form a baryon, and then the nonaquark state consequently decays into three baryons. We show this mechanism in the Fig5.

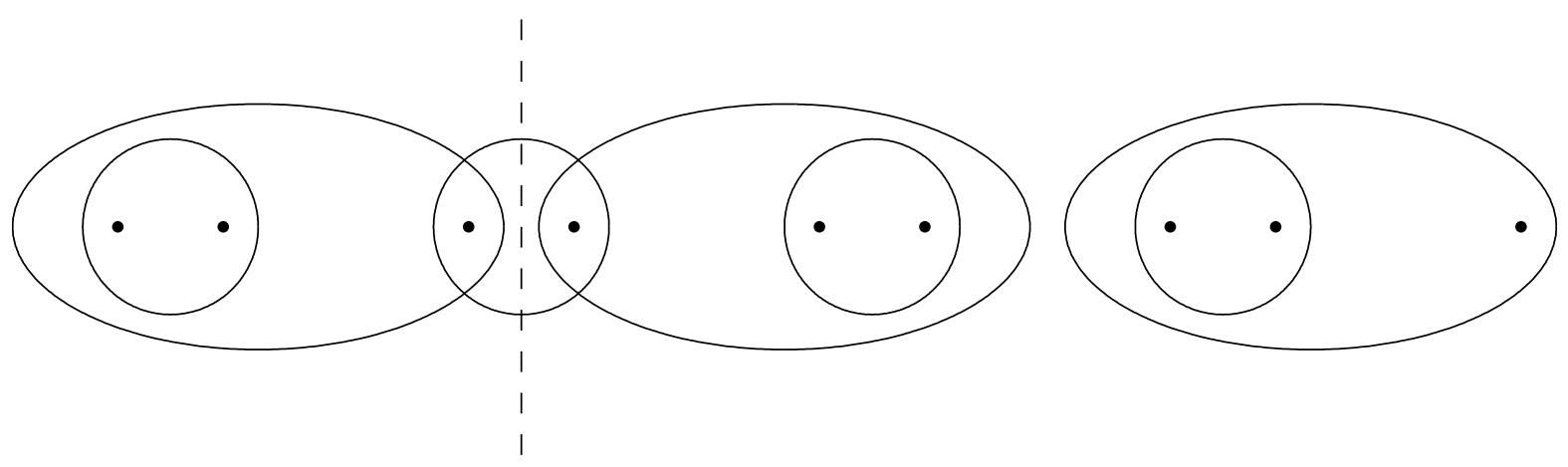

FIG. 5: The decay mechanism of Nonaquark states .

To the baryon octet, its quark content and the corresponding tensor are well-known, which is listed in the Table I. The tensor basis of the baryon octet is

$$
B_{r}^{l}=\frac{1}{\sqrt{3}} \varepsilon^{l m n} S_{r n} q_{m}
$$

with $S_{r n}=\frac{1}{\sqrt{2}}\left(q_{r} q_{n}+q_{n} q_{r}\right)$. And the baryon singlet $\Lambda_{1}^{0}$ is given by

$$
\begin{aligned}
\Lambda_{1}^{0} & =\frac{1}{2} \frac{1}{\sqrt{6}} \varepsilon^{k l m}\left(q_{l} q_{m}-q_{m} q_{l}\right) q_{k} \\
& =\frac{1}{\sqrt{6}}([d, s] u+[s, u] d+[u, d] s)
\end{aligned}
$$

From these wave function we can see:

$$
\left\{\begin{array}{l}
{[\mathrm{d}, \mathrm{s}] u=\Sigma^{0}+\frac{\sqrt{6}}{3} \Lambda_{1}^{0}-\frac{\sqrt{3}}{3} \Lambda_{8}^{0}} \\
{[\mathrm{~s}, \mathrm{u}] d=-\Sigma^{0}+\frac{\sqrt{6}}{3} \Lambda_{1}^{0}-\frac{\sqrt{3}}{3} \Lambda_{8}^{0}} \\
{[\mathrm{u}, \mathrm{d}] s=\frac{\sqrt{6}}{3} \Lambda_{1}^{0}+\frac{2 \sqrt{3}}{3} \Lambda_{8}^{0}}
\end{array}\right.
$$


TABLE I: Baryon octet and its' flavor wave function

\begin{tabular}{|c|c|c|}
\hline \hline State & Tensor & Quark content \\
\hline $\mathrm{p}$ & $B_{1}^{3}$ & $\frac{1}{\sqrt{2}}[\mathrm{u}, \mathrm{d}] \mathrm{u}$ \\
\hline $\mathrm{n}$ & $B_{2}^{3}$ & $\frac{1}{\sqrt{2}}[\mathrm{u}, \mathrm{d}] \mathrm{d}$ \\
\hline$\Sigma^{+}$ & $B_{1}^{2}$ & $\frac{1}{\sqrt{2}}[\mathrm{~s}, \mathrm{u}] \mathrm{u}$ \\
\hline$\Sigma^{0}$ & $\frac{1}{\sqrt{2}}\left(B_{1}^{1}-B_{2}^{2}\right)$ & $\left.\frac{1}{2}([\mathrm{~d}, \mathrm{~s}] \mathrm{u}+[\mathrm{u}, \mathrm{s}] \mathrm{d}]\right)$ \\
\hline$\Sigma^{-}$ & $B_{2}^{1}$ & $\frac{1}{\sqrt{2}}[\mathrm{~d}, \mathrm{~s}] \mathrm{d}$ \\
\hline$\Lambda^{0}$ & $-\frac{3}{\sqrt{6}} B_{3}^{3}$ & $\frac{1}{\sqrt{12}}(2[\mathrm{u}, \mathrm{d}] \mathrm{s}-[\mathrm{d}, \mathrm{s}] \mathrm{u}-[\mathrm{s}, \mathrm{u}] \mathrm{d})$ \\
\hline$\Xi^{-}$ & $B_{3}^{1}$ & $\frac{1}{\sqrt{2}}[\mathrm{~d}, \mathrm{~s}] \mathrm{s}$ \\
\hline$\Xi^{0}$ & $B_{3}^{2}$ & $\frac{1}{\sqrt{2}}[\mathrm{~s}, \mathrm{u}] \mathrm{s}$ \\
\hline \hline
\end{tabular}

\section{A. The decay of 27-plet}

Since the 27-plet is degenerate, i.e., $27_{1}, 27_{2}$ and $27_{3}$ are the three irreducible representatives of 27-dimension, we discuss the decays for each case following the method of dealing with the "fall-apart" decay [10]. Starting with the wave function of $S_{27_{1}, 1}^{+}$( or $S_{27_{1}, 1}^{0}$ etc.) which is given in Eq.(26) (Eq.(27) etc.), we rewrite the wave function in the form of (qqq)(qqq)(qqq) which is similar to Ref. 10], then using Eq. (56) and Table I and considering fermion statistics, we can map $S_{27_{1}, 1}^{+}$( or $S_{27_{1}, 1}^{0}$ etc.) into the ground state of three baryons, from which we can learn what particles are the decay products of the $S_{27_{1}, 1}^{+}$(or $S_{27_{1}, 1}^{0}$ etc.), and derive further the ratios of the branch fractions of these channels. We discuss the decays for each case respectively by means of the above spirit of dealing with the decays in the follows:

1. The first case: The wave function of $S_{27_{1}, 1}^{+}$and $S_{27_{1}, 1}^{0}$ is given by Eq.(26) and Eq.(27) separately, and they are mapped into

$$
\begin{gathered}
S_{27_{1}, 1}^{+} \rightarrow \frac{1}{\sqrt{2}} \Sigma^{0} p n \\
S_{27_{1}, 1}^{0} \rightarrow \frac{1}{2}\left(\sqrt{2} \Sigma^{-} p n+\frac{\sqrt{6}}{3} \Lambda_{1}^{0} n n+\frac{2 \sqrt{3}}{3} \Lambda_{8}^{0} n n\right) .
\end{gathered}
$$


Then, we obtain that the main decay channel of $S_{27_{1}, 1}^{+}$is $\Sigma N N$, and the ratio of coupling constants is

$$
g\left(S_{27_{1}, 1}^{+} \Sigma^{-} p n\right): g\left(S_{27_{1}, 1}^{+} \Lambda_{1}^{0} n n\right): g\left(S_{27_{1}, 1}^{+} \Lambda_{8}^{0} n n\right)=\sqrt{2}: \frac{\sqrt{6}}{3}: \frac{2 \sqrt{3}}{3} .
$$

Considering phase space effect, we get

$$
\frac{B R\left[S_{27_{1}, 1}^{+} \rightarrow \Sigma N N\right]}{B R\left[S_{27_{1}, 1}^{+} \rightarrow \Lambda N N\right]}=\frac{2}{\left.\left(\left(\frac{\sqrt{6}}{3}\right)^{2}+\frac{2 \sqrt{3}}{3}\right)^{2}\right) \times 4.164} \approx 0.24
$$

Therefore, $S_{27_{1}, 1}^{0}$ dominantly decays into $\Lambda N N$, and $S_{27_{1}, 1}^{0}$ can not be $S^{0}(3115)$.

2. The second case: Eq.(30) and Eq.(31) give the wave function of $S_{27_{2}, 1}^{+}$and $S_{27_{2}, 1}^{0}$ in this case, and the map is as following

$$
\begin{gathered}
S_{27_{2}, 1}^{+} \rightarrow \frac{1}{2 \sqrt{2}}\left(\Sigma^{+} n n+\Sigma^{-} p p+\sqrt{2} \Sigma^{0} p n\right), \\
S_{27_{2}, 1}^{0} \rightarrow \frac{1}{2 \sqrt{2}}\left(-\Sigma^{0} n n+\sqrt{2} \Sigma^{-} p n-\sqrt{6} \Lambda_{1}^{0} n n-\sqrt{3} \Lambda_{8}^{0} n n\right) .
\end{gathered}
$$

We note that in this case $S_{27_{2}, 1}^{+}$also can only decay into $\Sigma N N$, and the ratio of the branch fractions

$$
B R\left[S_{27_{2}, 1}^{+} \rightarrow \Sigma^{+} n n\right]: B R\left[S_{27,1}^{+} \rightarrow \Sigma^{-} p p\right]: B R\left[S_{27,1}^{+} \rightarrow \Sigma^{0} p n\right]=1: 1: 2
$$

and the main decay modes of $S_{27_{2}, 1}^{0}$ is $\Lambda N N$

$$
\frac{B R\left[S_{27_{2}, 1}^{0} \rightarrow \Sigma N N\right]}{B R\left[S_{27_{2}, 1}^{0} \rightarrow \Lambda N N\right]} \approx 0.08
$$

3. The third case: The wave function of $S_{27_{3}, 1}^{+}$and $S_{27_{3}, 1}^{0}$ is given by Eq.(34) and Eq.(35), similarly

$$
\begin{gathered}
S_{27_{3}, 1}^{+} \rightarrow \frac{3}{2} \Sigma^{0} p n+\frac{1}{2 \sqrt{2}} \Sigma^{+} n n+\frac{1}{2 \sqrt{2}} \Sigma^{-} p p \\
S_{27_{3}, 1}^{0} \rightarrow \frac{1}{4 \sqrt{2}}\left(6 \sqrt{2} \Sigma^{-} p n-2 \Sigma^{0} n n-\frac{2 \sqrt{6}}{3} \Lambda_{1}^{0} n n+\frac{2 \sqrt{3}}{3} \Lambda_{8}^{0} n n\right) .
\end{gathered}
$$

From the above two formula, we know that in this case $S_{27_{3}, 1}^{+}$decay mainly into $\Sigma N N$ not to $\Lambda N N$, and

$$
B R\left[S_{27_{3}, 1}^{+} \rightarrow \Sigma^{0} p n\right]: B R\left[S_{27_{3}, 1}^{+} \rightarrow \Sigma^{+} n n\right]: B R\left[S_{27_{3}, 1}^{+} \rightarrow \Sigma^{-} p p\right]=18: 1: 1
$$

and the ratio of branch fractions

$$
\frac{B R\left[S_{27_{3}, 1}^{0} \rightarrow \Sigma N N\right]}{B R\left[S_{27_{3}, 1}^{0} \rightarrow \Lambda n n\right]} \approx 2.8
$$

We note that in this case the main decay mode of $S_{27_{3}, 1}^{0}$ is $\Sigma N N$ which is consistent with the decay of $S^{0}(3115)$. 


\section{B. The decay of $\overline{35}$-plet}

Starting with the wave function of $S_{35,1}^{+}$which is given in Eq.(42), we discuss the decays by means of the method used in the previous sub-section to deal with the decays. We map $S \frac{+}{35,1}$ into the ground state of three baryons

$$
S_{\frac{+}{35,1}}^{+} \rightarrow \frac{1}{2}\left(\Sigma^{-} p p+\Sigma^{+} n n\right)
$$

So, $S_{\overline{35,1}}^{+}$can only decay into $\Sigma^{-} N N$, but can not decay into $\Lambda^{-} N N$ (N stands for nucleon,i.e., proton or neutron). And the ratio of branch fractions is

$$
\frac{B R\left[S_{\frac{+}{35,1}}^{+} \rightarrow \Sigma^{-} p p\right]}{B R\left[S_{\overline{35}, 1}^{+} \rightarrow \Sigma^{+} n n\right]}=\frac{1}{1}=1
$$

Similarly $S \frac{+}{35}$ whose wave function is defined in Eq.(43) is mapped onto

$$
S_{\frac{+}{35}} \rightarrow \frac{1}{\sqrt{6}}\left(\Sigma^{-} p p-\Sigma^{+} n n\right)
$$

also $S \frac{+}{35}$ decay only into $\Sigma^{-} N N$, can not decay into $\Lambda^{-} N N$, and the ratio of branch fractions is

$$
\frac{B R\left[S_{\overline{35}}^{+} \rightarrow \Sigma^{-} p p\right]}{B R\left[S_{\overline{35}}^{+} \rightarrow \Sigma^{+} n n\right]}=\frac{1}{1}=1
$$

We also obtain that the $S_{\frac{+}{35}}^{+} \Sigma^{-} p p$ and $S_{\frac{+}{35}} \Sigma^{+} n n$ interactions have different phase, while the $S_{\frac{1}{35,1}}^{+} \Sigma^{-} p p$ and $S_{35,1}^{+} \Sigma^{+} n n$ interactions have the same phase (see Eqs.(171) and (69)).

In the same way, starting from Eq.(41) which is the wave function of $S \frac{0}{35,1}$, we have:

$$
S \frac{0}{35,1} \rightarrow \frac{1}{2}\left(-\Sigma^{0}+\sqrt{3} \Lambda_{8}^{0}\right) n n
$$

So the main decay channel of $S \frac{0}{35,1}$ is $\Lambda N N$, but not $\Sigma N N$, i.e., ratio of the effective couplings reads

$$
\frac{g\left(S_{\overline{35,1}}^{0} \Sigma^{0} n n\right)}{g\left(S_{\overline{35}, 1}^{0} \Lambda_{8}^{0} n n\right)}=-\frac{1}{\sqrt{3}} .
$$

Considering the different three body phase space, we can obtain the the ratio of branch fractions

$$
\frac{B R\left[S \frac{0}{35,1} \rightarrow \Sigma^{0} n n\right]}{B R\left[S \frac{0}{35,1} \rightarrow \Lambda_{8}^{0} n n\right]} \approx 0.05 .
$$

So the observed $S^{0}(3115)$ 1] can not be $S \frac{0}{35,1}$. 
From the decay of $\overline{\mathbf{3 5}}$-plet and $\mathbf{2 7}$-plet, we see that the nonaquark state $S^{0}(3115)$ can only possibly belong to 27-plet, and its flavor configuration is Eq.(35), or its main component is $S_{27_{3}, 1}^{0}$ with small mixing of $S_{27_{1}, 1}^{0}, S_{27_{2}, 1}^{0}, S_{35,1}^{0}$. While $S^{+}(3140)$ maybe belong to $\overline{\mathbf{3 5}}$-plet or 27-plet, it possibly is $S_{27_{1}, 1}^{+}, S_{27_{2}, 1}^{+}, S_{27_{3}, 1}^{+}, S_{35,1}^{+}$or the mixing of all of them, since these states have same quantum numbers. From the discussion of subsection A in section II , we know that in the exact $S U(3)^{\text {flavor }} \times S U(3)^{\text {color }} \times S U(2)^{\text {spin }}$ limit, $S^{0}(3115)$ and $S^{+}(3140)$ possibly belong to 27 -plet, this further give support to our suggestion that $S^{0}(3115)$ can only possibly belong to 27 -plet. Furthermore in this case the nonaquark states have negative parity, this is a unusual results, since "standard" nonaquark state which involve 9 quarks in relative S-wave have positive parity, there maybe really exists exotica nonaquark $\mathbf{2 7}$-plet. We can obtain useful information about $S^{+}(3140)$ by experimentally measuring the branch fractions of its decay channels.

\section{DISCUSSION AND CONCLUSION}

In summary, we have obtain the wave functions of the nonaquark states in $S U(3)$ quark model with diquark correlation using standard direct tensor decomposition, we predict the existence of other nonaquark states. It would be helpful for constructing the effective interaction Lagrangian to describe the nonaquark decays with rational $S U(3)$-flavor structure. We obtain some interesting mass sum rules for the nonaquark $\overline{35}$-plet and 27 -plet, but it is still open to fix the spectrum of $\overline{\mathbf{3 5}}$-plet and $\mathbf{2 7}$-plet due to the scarcity of experiments. More data are expected. Under the assumption of the "fall-apart" decay mechanism which has been subtly used in studying pentaquark decays, we find out that the $S^{0}(3115)$ belongs to a 27-plet. Its main component is $S_{273,1}^{0}$, and the mixing with $S_{27_{1}, 1}^{0}, S_{27_{2}, 1}^{0}, S_{35,1}^{0}$ must be few. It is possible that $S^{0}(3115)$ and $S^{+}(3140)$ belong to the same isospin multiplet, since their mass difference is about $25 \mathrm{MeV}$ which can be interpreted by electromagnetic interaction and u,d quark mass difference. Further in the exact $S U(3)^{\text {flavor }} \times S U(3)^{\text {color }} \times S U(2)^{\text {spin }}$ limit, $S^{0}(3115)$ and $S^{+}(3140)$ are belong to the $\mathbf{2 7}$-plet, and its parity is negative. We suggest to study the decays of $S^{+}(3140)$ more in experiment, especially the ratios of the various decay mode's branch fractions, through which we can learn the structure of $S^{+}(3140)$ and maybe discover new nonaquark state .

There maybe other types of quark correlation in nonaquark state, such as (qqq)-(qqq)- 


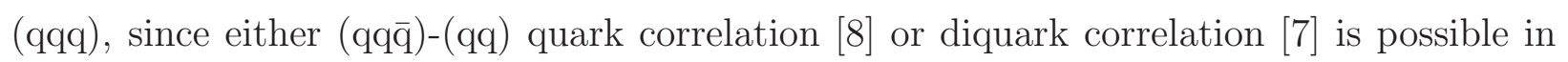
pentaquark, they have different flavor and color structures from the diquark correlation, so the masses, the decay property, and the products etc of the states may be different from the prediction of quark model with diquark correlation.

It would be interesting to study the mixing between the nonaquark states with same quantum numbers. It is necessary to considering the mixing in order to compare theoretical prediction with experimental data more precisely. This would be highly nontrivial.

Finally, we might image that this multi-baryon problem could be studied in other quark models, e.g., the model in [19], and in the chiral soliton model [20].

\section{ACKNOWLEDGMENTS}

We acknowledge Dr. J. Deng for his help in plotting the diagrams. We would like also to thank the referee for his suggestion to discuss the color symmetry in the nonaquark wavefunction. This work is partially supported by National Natural Science Foundation of

China under Grant Numbers 90403021, and by the PhD Program Funds of the Education Ministry of China and KJCX2-SW-N10 of the Chinese Academy.

[1] T.Suzuki, H.Bhang et al.,PLB597 (2004),263.

[2] M.Iwasaki,T.Suzuki et al., nucl-ex/0310018.

[3] Y.Akaishi and T.Yamazaki,Phys.Rev.C 65 (2002)044005.

[4] Y.Maezawa,T.Hatsuda, S.Sasaki hep-ph/0412025.

[5] A.Dote, H.Horiuchi, Y.Akaishi,Phys.Rev.C70:044313,2004 nucl-th/0309062.

[6] J.Mares, E.Friedman, Avraham Gal,Phys.Lett.B606:295-302,2005 nucl-th/0407063.

[7] R.L.Jaffe and F.Wilcek,Phys.Rev.Lett 91,232003(2003)hep-ph/0307341.

[8] M.Karliner,H.J.Lipkin, hep-ph/0307243.

[9] M.Anselmino,E.Predazzi,S.Ekelin,S.Fredriksson and D.B.Lichtenberg,Rev.Mod.Phys.65,(1993)1199.

[10] F.E. Close, J.J. Dudek,Phys.Lett. B586 (2004) 75-82 hep-ph/0401192

[11] F.E.Close,Conference Summary talk in Proceedings of Hadrons03 hep-ph/0311087.

[12] F.E.Close, An Introduction to Quarks and Partons (Academic Press,London,1970).

[13] Yongseok Oh, Hungchong Kim,Phys.Rev. D70 (2004)094022 hep-ph/0405010. 
[14] F.E.Low, Symmetries and Elementary Paricles (Gordon and Breach,New York,1967).

[15] Fayyazuddin and Riazuddin, A modern Introduction to Particles Physics (World Scientific,Singapore,1992).

[16] B.Jennings, K.Maltman,Phys.Rev. D69 (2004) 094020, hep-ph/0308286.

[17] C.E.Carlson, C.D.Carone etal.,Phys.Rev. D70 (2004) 037501 hep-ph/0312325.

[18] F.Buccella, P.Sorba,Mod.Phys.Lett. A19 (2004) 1547,hep-ph/0401083.

[19] A.T.M.Aerts,P.L.G.Mulders and J.J.de Swart,Phys.Rev.D 17 (1978)260.

[20] C.L.Schat, N.N.Scoccola,Phys.Rev. D62 (2000) 074010 hep-ph/0003247

[21] A. De Rujula, H. Georgi and S. L. Glashow, Phys. Rev. D 12, 147 (1975).

[22] T. DeGrand, R. L. Jaffe, K. Johnson and J. E. Kiskis, Phys. Rev. D 12, 2060 (1975).

[23] G.'t Hooft, Phys. Rev. D 14, 3432 (1976) [Erratum-ibid. D 18, 2199 (1978)].

[24] E.V.Shuryak, Nucl. Phys. B203,93(1982); T. Schafer and E. V. Shuryak, Rev. Mod. Phys. 70, 323 (1998); E. Shuryak and I. Zahed, Phys. Lett. B 589, 21 (2004) arXiv:hep-ph/0310270.

[25] C.Itzykson, M.Nauenberg, Rev.Mod.Phys.38:95-120,1966. 


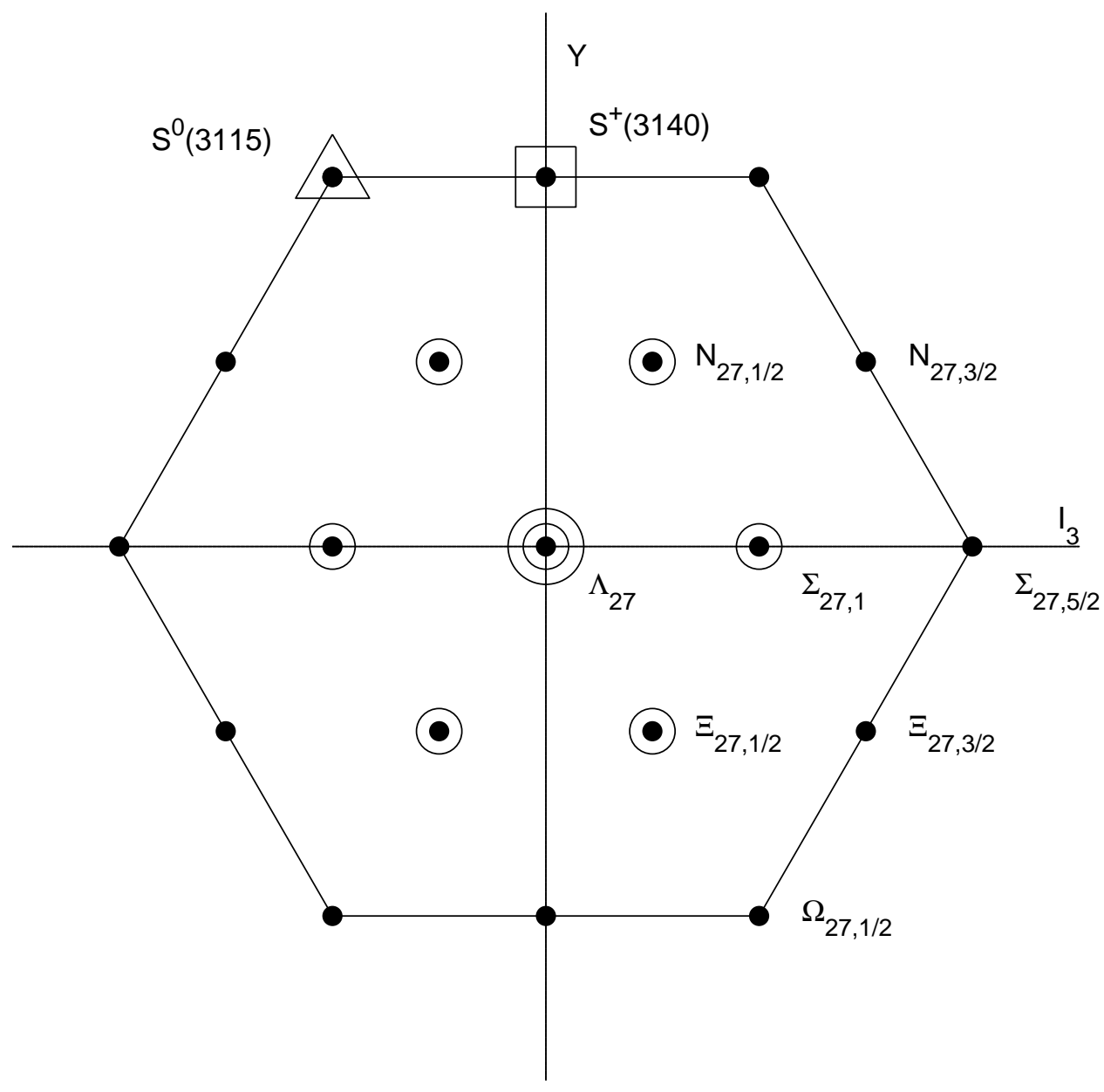

FIG. 6: The weight diagram of nonaquark 27-plet, and the location of $S^{0}(3115)$ and $S^{+}(3140)$ are expressly shown by the triangle and square respectivelly 


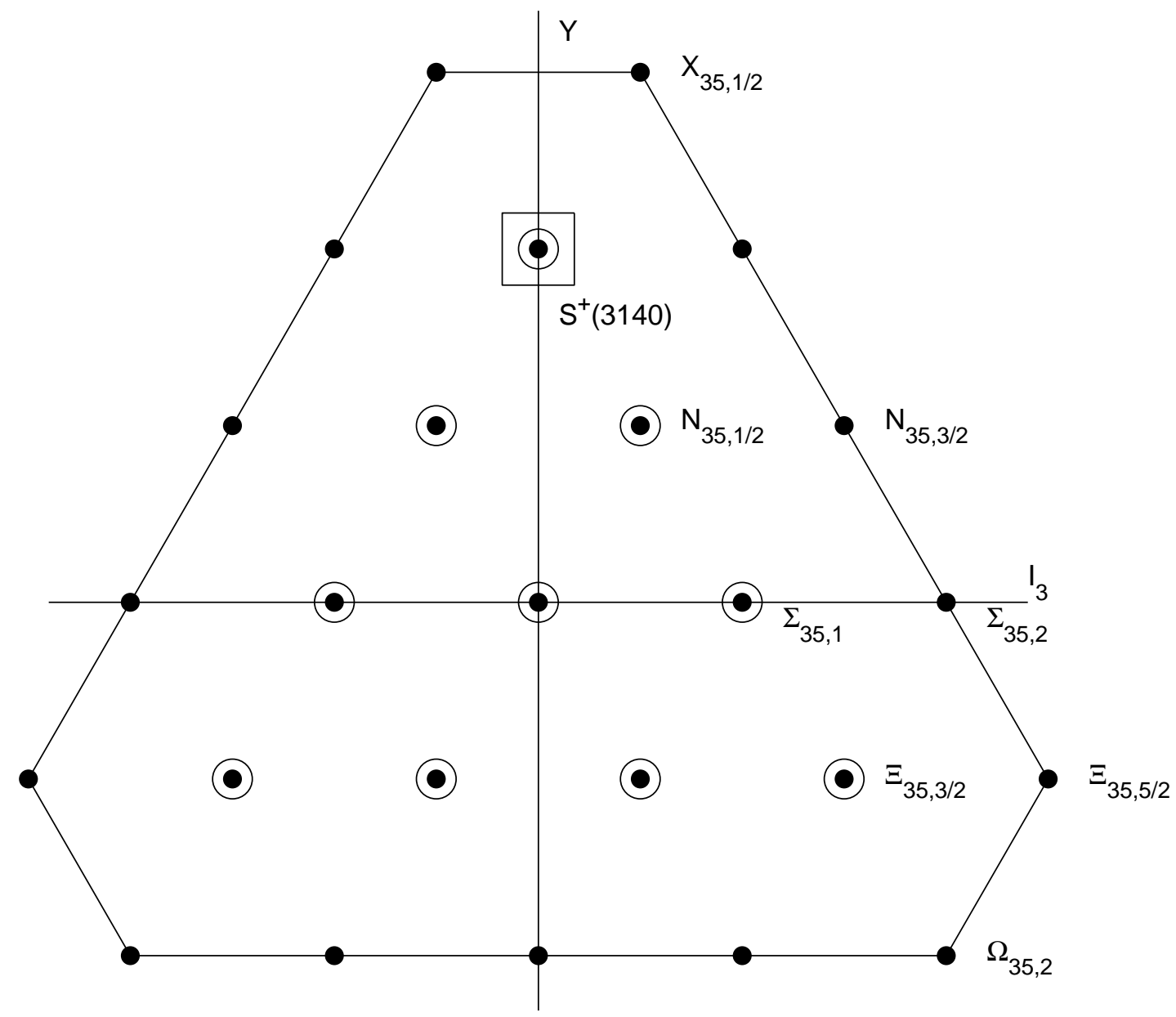

FIG. 7: The weight diagram of nonaquark $\overline{\mathbf{3 5}}$-plet, only $S^{+}(3140)$ is specially shown by square, whereas $S^{0}(3115)$ is not shown. Because our analysis indicate that $S^{0}(3115)$ can not belong to $\overline{35}$-plet 